10. - On a definition of the integral in topological spaces. I, II, Casopis Pest. Mat. 89 (1964), 129-147, 257-277.

11. - A note on the lower derivate of a set function and semihereditary systems of sets, Proc. Amer. Math. Soc. 18 (1967), 1020-1025.

12. - On the lower derivate of a set function, Canad. J. Math. 20 (1968), 1489-1498.

13. - The integral in topological spaces. I and II (to appear).

14. - Singular integrals are Perron integrals of a certain type (to appear).

15. W. F. Pfeffer and W. J. Wilbur, $A$ note on cluster points of a semihereditary stable system of sets, Proc. Amer. Math. Soc. 21 (1969).

16. - On the measurability of Perron integrable functions (to appear).

17. W. J. Wilbur, On non-absolute integration in topological spaces, Ph.D. thesis, University of California, Davis, Calif.

18. S. Saks, Theory of the integral, Hafner, New York, 1937.

University of California, Davis, California 95616

\title{
ON GENERALIZED COMPLETE METRIC SPACES
}

BY JAMES D. STEIN, JR.

Communicated by J. B. Diaz, October 3, 1968

The following remarks are of interest in connection with the research announcement [1]:

Lemma. A generalized metric space is the disjoint union of metric spaces such that each metric space is infinitely distant from every other metric space.

Proof. Note that $d(x, y)<\infty$ is an equivalence relation, and the equivalence classes obtained are metric spaces. Also, if the generalized space is complete, so is each metric space. Q.E.D.

Let $M=\mathrm{V}_{\alpha e A} M_{\alpha}$ denote the above partitioning. The Banach contraction principle becomes

Proposition 1. Let $T$ be a strict contraction of a generalized complete metric space $M=V_{\alpha \in A} M_{\alpha}, 0 \leqq q<1, d(x, y)<\infty \Rightarrow d(T x, T y) \leqq q d(x, y)$. For each $\alpha \in A, \exists \beta \in A$ such that $T\left(M_{\alpha}\right) \subseteq M_{\beta}$. There is a unique periodic point of order $n$ in each $M_{\alpha}$ such that $T^{n}\left(M_{\alpha}\right) \subseteq M_{\alpha}$.

Proof. Let $x, y \in M_{\alpha}, T x \in M_{\beta}$. Then $d(x, y)<\infty \Rightarrow d(T x, T y)<\infty$ $\Rightarrow T y \in M_{\beta}$. Since $T^{n}$ is a strict contraction of the complete metric space $M_{\alpha}$, it has a unique fixed point, which is a periodic point of order $n$ for T. Q.E.D.

The local contraction principle becomes 
Proposition 2. Let $T$ be a local contraction $(d(x, y) \leqq C \Rightarrow d(T x, T y)$ $\leqq q d(x, y))$ of a complete generalized metric space. For each $\alpha \in A, x, y$ $\in M_{\alpha}$, define $x \sim y$ iff $\exists x_{0}, \cdots, x_{n} \in M_{0}$ such that $x=x_{0}, y=x_{n}$, $d\left(x_{i}, x_{i+1}\right) \leqq C$ for $0 \leqq i \leqq n-1$. Then $\sim$ is an equivalence relation on each $M_{\alpha}$; call the equivalence classes thus obtained $C$-components. $T$ maps each $C$-component of $M_{\alpha}$ into a $C$-component of some $M_{\beta}$. There is a unique periodic point of order $n$ in each C-component $N$ of $M_{\alpha}$ such that $T^{n}(N) \subseteq N$.

Proof. Clearly $x \sim y$ is an equivalence relation; and if $x, y \in M_{\alpha}$ and $x \sim y$, let $x=x_{0}, \cdots, x_{n}=y$ be the chain. Then $d\left(T x_{i}, T x_{i+1}\right)$ $\leqq q d\left(x_{i}, x_{i+1}\right) \leqq C$, and so $T x \sim T y$ in some $M_{\beta}$. The remainder is Theorem 1.4 of Bonsall (or Edelstein) of On some fixed point theorems of functional analysis. Q.E.D.

Several of Edelstein's and Rakotch's results go over analogously.

\section{REFERENCE}

1. J. B. Diaz and Beatriz Margolis, A fixed point theorem of the alternative, for contractions on a generalized complete metric space, Bull. Amer. Math. Soc. 74 (1968), 305-309.

University of California, Los Angeles, California 90024 